\title{
EXAMEN DE CALIDAD DE LA EDUCACIÓN SUPERIOR SABER PRO: FACTORES SOCIODEMOGRÁFICOS EN EL DESEMPEÑO ACADÉMICO EN INSTRUMENTACIÓN QUIRÚRGICA
}

\author{
Sandra Giovanna Cortés *, César A rcadio Piñeros **
}

\section{Resumen}

Los examenes de calidad de la educación superior Saber Pro en Colombia hacen parte de la estrategia gubernamental para garantizar la alta calidad de la educación. El programa de instrumentación quirúrgica lo ha presentado desde 2010; se desarrolla en 16 instituciones de educación superior (IES) del país. Objetivo: determinar los factores sociodemográficos asociados con el desempeño entre 2010 y 2011. Métodos: análisis multinivel para determinar la correlación de las variables sociodemográficas e institucionales con las académicas mediante la información de la base de datos FTP del ICFES. El análisis se hizo con medida de tendencia central, dispersión y frecuencias absolutas. Se construyó una base de datos en excel y se utilizó el programa stata 12. Resultados: el principal factor asociado con el desempeño en los componentes genéricos del examen Saber Pro 2010 y 2011 es la edad más joven del estudiante, en los demás componentes existen diferentes factores de acuerdo con la competencia evaluada.

Palabras clave: instrumentación quirúrgica; factores sociodemográficos, examen de calidad de la educación superior, Saber Pro.

Abreviaturas: IES, institución de educación superior.

\section{TEST ON QUALITY OF HIGHER EDUCATION SABER PRO: SOCIODEMOGRAPHIC FACTORS ASSOCIATED WITH RESULTS OBTAINED BY SURGICAL INSTRUMENT TECHNICIANS}

\section{Abstract}

\begin{abstract}
The tests on the quality of undergraduate education in Colombia (Saber Pro) are part of the strategy undertaken by the National Government to guarantee the high quality of education. The surgical instrument technician program students have taken this exam since 2010. It is applied in 16 Colombian undergraduate education institutions and although they share the objective of formation the test results may be associated to various factors, some institution-related and others related to the student's social and demographic characteristics. Objective: to determine the social and demographic characteristics associated to test results between 2010 and 2011 . Methods: a multilevel analysis to determine the correlation of social and demographic characteristics and institutional variables with academic variables using the information recorded in the ICFES FTP data bases. Students of Colombian instrument technician schools and programs were included. Cases with incongruent information were excluded. Central tendency measures, dispersion and absolute frequencies were used. An excel data base was constructed and the Stata 12 program was utilized. Results: younger age of the student was the major result-associated factor in the generic components of the 2010 and 2011 Saber Pro exam. Other components are associated with various factors related to the specific competence assessed.
\end{abstract}

Key words: surgical instrument technician; social and demographic factors, test on quality of higher education, Saber Pro

Fecha recibido: diciembre 19 de 2014 - Fecha aceptado: marzo 12 de 2015

* Profesor Asociado, Fundación Universitaria de Ciencias de la Salud, Bogotá DC, Colombia
** Instructor Asistente, División de Investigaciones, Fundación Universitaria de Ciencias de la Salud, Bogotá DC, Colombia 


\section{Introduc ción}

La evaluación de las competencias adquiridas durante el proceso de enseñanza aprendizaje es una tendencia mundial, la cual fue adoptada en Colombia a través de la creación del examen de calidad de la educación superior. Hasta 2009 se denominó ECA ES, su objetivo principal fue comprobar el grado de desarrollo de las competencias de los estudiantes que cursan el último año de los programas académicos de pregrado., L os resultados obtenidos fueron una fuente importante de información para las universi dades, que los utilizaron para conocer el comportamiento académico de sus alumnos frente a otras pruebas de estado o bien las específicas realizadas por las IES; sin embargo en algunos casos los estudiantes afirmaron que el examen es más un requisito para graduarse y un mecanismo para la jerarquización de las universidades, ${ }^{3,4}$ desviándose de su objetivo principal y generando fuertes críticas en el ámbito educativo. En consecuencia y gracias a la actualización legislativa de la evaluación externa real izada en 2009 con la promulgación de la ley 1324 y el decreto 3963, se reglamentó la obligatoriedad del examen y se reorganizó su estructura, en la cual se busca evaluar las competencias genéricas necesarias para el adecuado desempeño profesional, independiente del programa que hayan cursado y las competencias específicas, teniendo en cuenta los elementos disciplinares fundamental es de la formación superior, que son comunes a grupos de programas en una o más áreas del conocimiento5-7

El examen de calidad de la educación superior para el programa de instrumentación quirúrgica fue construido por el Instituto Colombiano para la Evaluación de la Educación (ICFES) y la A sociación Colombiana de Facultades de Instrumentación Quirúrgica (ACFIQ) fundamentado en el objeto de estudio de la profesión. Se incluyeron los componentes social-humanísticos e investigativo, procesos quirúrgicos, control de infecciones, inglés y comunicación oral. Esta estructura se mantuvo de 2006 a $2009^{8}$, durante este periodo la Fundación U niversitaria de Ciencias de la Salud (FUCS) realizó un estudio para establecer correlación entre el desempeño académico y los resultados obtenidos por los estudiantes en las diferentes pruebas de estado, análisis que hizo parte de las estrategias de seguimiento y evaluación permanente de la calidad educativa del programa. ${ }^{9}$

Durante la etapa de transición del examen de evaluación de competencias específicas a genéricas, comprendida entre 2010 y 2011, el ICFES construyó y aplicó las pruebas Saber Pro, las cuales incluían los módulos de pensamiento crítico, solución de problemas, entendimiento interpersonal y escritura, bajo esta modalidad presentaron el examen 1.138 estudiantes de instrumentación quirúrgica de distintas IES a nivel nacional. A unque los programas comparten un objetivo de formación, existen factores sociodemográficos como son las características individuales, sociales, familiares y de la institución, que pueden aportar al desarrollo de las habilidades de pensamiento y comunicación de los estudiantes, las cuales se consideran esenciales para un adecuado desempeño profesional y académico, y constituyen la base de la evaluación por competencias en los módulos del examen Saber Pro.

En países de Latinoamérica y el Caribe se destacan como determinantes esenciales del rendimiento académico las características socioeconómicas y el nivel educativo de la familia; en el caso de Colombia la situación es similar, ya que diversos estudios demuestran que los factores socioeconómicos familiares afectan tanto el rendimiento académico de un estudiante en los diferentes niveles de educación, como en los resultados obtenidos en las pruebas ICFES presentadas al final de sus estudios de educación secundaria. ${ }^{10-12}$ Este tema se ha abordado en diferentes programas de educación superior, es el caso del de economía, para el cual en 2004 se realizó un análisis multinivel con datos de 2.377 estudiantes de 52 universidades en todo el país, que demostró la existencia de diferencias significativas entre los puntajes medios de las universidades y los obtenidos por los estudiantes, que se explican por las características personales y las de la institución a la que pertenecen. ${ }^{13}$ Este trabajo pretende determinar los factores sociodemográficos de los estudiantes de instrumentación quirúrgica, asociados con el desempeño en el examen de calidad de educación superior Saber Pro entre 2010 y 2011 a nivel nacional. 


\section{Materiales y métodos}

La información se obtuvo a partir de la base de datos FTP del ICFES disponible para el público. Se incluyeron estudiantes de las facultades y programas de instrumentación quirúrgica del país, se excluyeron aquellos con datos inconsistentes. Se tuvieron en cuenta las variables sociodemográficas, académicas e institucionales, las cuales fueron analizadas con frecuencias absolutas, medidas de tendencia central y de dispersión. Después se ajustó un modelo multinivel para determinar la asociación de las variables sociodemográficas e institucionales con las académicas, en el cual se estableció como variable dependiente el resul tado de cada una de los componentes del examen. $L$ as variables independientes fueron las características sociodemográficas e institucionales. El análisis estadístico de la información se realizó en stata 12. Este trabajo fue avalado por el comité de ética de investigación con seres humanos del Hospital de San J osé y de la Fundación U niversitaria de Ciencias de la Salud, Bogotá DC, Colombia.

\section{Resultados}

Durante el periodo 1.116 estudiantes de instrumentación quirúrgica en el país presentaron el examen. Las características generales de la población y los factores sociodemográficos considerados para el estudio se presentan en la Tabla 1, discriminados por año. En la Tabla 2 se describe el carácter académico, el origen y el número de estudiantes que presentaron el examen. Después de ajustar el modelo multinivel se encontraron los siguientes factores asociados con el desempeño académico del estudiante, para cada uno de los componentes del examen.

El componente de lectura crítica y comprensión lectora se asoció solo con el factor edad. La comunicación escrita con el sexo femenino, el pago de la matrícula asumido por los padres, el crédito educativo y tener computador. El razonamiento cuantitativo con el pago de la matrícula asumido por los padres. El componente de inglés se asoció con la edad del estudiante, el estrato socioeconómico, el acceso a internet desde el hogar y el nivel educativo superior alcanzado por el padre y la madre. El pensamiento crítico con la edad del estu- diante, el número de personas que conforman el grupo familiar, el estrato socioeconómico y el nivel educativo superior alcanzado por el padre. El entendimiento interpersonal con el sexo femenino, la edad del estudiante, el número de personas a cargo, el nivel educativo superior alcanzado por el padre y su ocupación independiente. La solución de problemas con la edad, el valor de la matricula superior a tres millones de pesos, el estrato socioeconómico, tener computador en el hogar y si el estudiante realiza al gún trabajo. En la Tabla 3 se detalla para cada componente del examen los factores asociados. El origen de la IES se asoció con el componente de comunicación escrita ( $p=0.029$ ). El valor de la matrícula del año anterior con pensamiento crítico ( $p=$ 0.023 ) y solución de problemas ( $p=0.002$ ).

\section{Discusión}

Se encontraron características del estudiante y de la IES relacionadas con los resultados en los diferentes componentes de la prueba, dentro de las características individuales del estudiante. La edad es el factor que más se asoció con los resultados obtenidos en los diferentes componentes, ya que los más jóvenes obtuvieron mejores resultados, lo cual está de acuerdo con estudios similares realizados en Colombia. ${ }^{14}$

A unque diversas investigaciones sobre la calidad de la educación en Colombia señalan que ser de sexo masculino favorece el desempeño académico tanto en los diferentes niveles de educación como en los resultados de las pruebas de estado Saber $11^{10,11}$, para el caso de instrumentación quirúrgica se evidenció un comportamiento que contrasta con la mayoría de estudios analizados, ya que en la comunicación escrita y el entendimiento interpersonal la influencia del sexo femenino en los resultados fue mayor, lo cual se podría explicar porque dichos componentes se enfocan en la competencia para comunicar ideas por escrito con base en el análisis de la información suministrada, así como la competencia para analizar y evaluar situaciones laborales e interpersonales, demostrando un buen nivel en la comprensión de sentimientos de otras personas ${ }^{15}$, características que se relacionan con mayor frecuencia con el sexo femenino y en nuestro caso, pueden explicar los mejores resultados que obtuvieron las mujeres en la prueba. 


\begin{tabular}{|c|c|c|c|c|}
\hline \multicolumn{5}{|c|}{$\begin{array}{r}\text { Tabla I. Características g } \\
\text { la población según el año de } \\
\text { de la prueba }\end{array}$} \\
\hline & \multicolumn{2}{|r|}{2010} & \multicolumn{2}{|c|}{2011} \\
\hline & $\mathbf{n}$ & (\%) & $\mathbf{n}$ & $(\%)$ \\
\hline Género - femenino & 452 & $(84,9)$ & 584 & $(85,2)$ \\
\hline Edad - promedio (DE) & 23,7 & $(4,6)$ & 23,4 & $(4,5)$ \\
\hline \multicolumn{5}{|l|}{ Estado civil } \\
\hline soltero & 459 & $(86,2)$ & 521 & $(89,2)$ \\
\hline casado & 42 & $(7,8)$ & 32 & $(5,48)$ \\
\hline Estudiantes cabeza de familia & 39 & $(7,3)$ & 33 & $(5,6)$ \\
\hline \multicolumn{5}{|l|}{$\mathbf{N}^{\circ}$ de personas a cargo } \\
\hline ninguna & 442 & $(83,0$ & 505 & $(86,4)$ \\
\hline uno & 52 & $(9.77)$ & 51 & $(8,7)$ \\
\hline dos & 20 & $(3,7)$ & 19 & $(3,2)$ \\
\hline \multicolumn{5}{|l|}{$\begin{array}{l}\mathbf{N}^{\circ} \text { de personas que confor- } \\
\text { man el hogar }\end{array}$} \\
\hline tres & 77 & $(14.4)$ & 114 & $(19,5)$ \\
\hline cuatro & 189 & $(35.5)$ & 175 & $(29,9)$ \\
\hline cinco & 109 & $(20.4)$ & $13 \mid$ & $(22,4)$ \\
\hline \multicolumn{5}{|l|}{$\begin{array}{l}\text { Estrato socioeconómico de } \\
\text { la vivienda actual }\end{array}$} \\
\hline uno & 74 & $(13.1)$ & 74 & $(12.6)$ \\
\hline dos & 184 & $(34.5)$ & 228 & (39) \\
\hline tres & 218 & $(40.9)$ & 216 & $(36.9)$ \\
\hline \multicolumn{5}{|l|}{ Situación laboral actual } \\
\hline $\begin{array}{l}\text { sí, para contribuir a pagar su } \\
\text { matrícula y gastos del hogar }\end{array}$ & 85 & $(15.9)$ & 62 & $(10.6)$ \\
\hline sí, para adquirir experiencia & 11 & $(2.0)$ & 22 & $(3.7)$ \\
\hline \multicolumn{5}{|l|}{$\begin{array}{l}\text { Bienes o servicios del hogar } \\
\text { habitual }\end{array}$} \\
\hline televisor a color & 413 & $(77.6)$ & 447 & $(76.5)$ \\
\hline computador & 396 & $(74.4)$ & 497 & $(85.1)$ \\
\hline conexión a internet & 276 & $(51.8)$ & 412 & $(70.5)$ \\
\hline automóvil particular & 125 & $(23.5)$ & 161 & $(27.5)$ \\
\hline \multicolumn{5}{|l|}{$\begin{array}{l}\text { Total de ingresos mensuales } \\
\text { del hogar habitual }\end{array}$} \\
\hline menos de I SML & 39 & (7.3) & 27 & $(4,6)$ \\
\hline entre I y menos de 2 SML* & 183 & $(34.4)$ & 237 & $(40.5)$ \\
\hline entre 2 y menos de 3 SML* & 191 & $(35.9)$ & 159 & $(27.2)$ \\
\hline entre 3 y menos de 5 SML* & 102 & $(19.19)$ & 113 & $(19.3)$ \\
\hline
\end{tabular}

* Salario Mínimo Legal en Colombia.

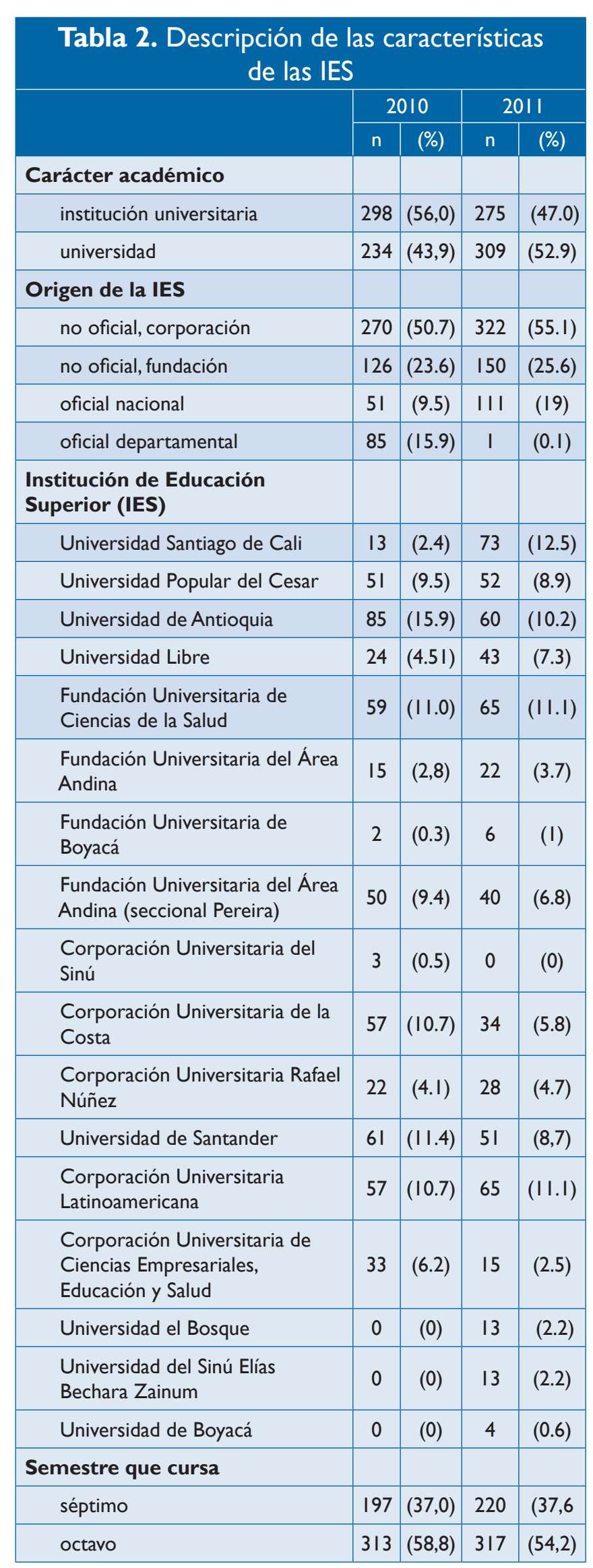




\begin{tabular}{|c|c|c|}
\hline & coeficiente & $\mathbf{p}$ \\
\hline \multicolumn{3}{|l|}{ Lectura crítica } \\
\hline edad & -0.032 & 0.00 \\
\hline \multicolumn{3}{|l|}{ Comunicación escrita } \\
\hline género & -0.728 & 0.01 \\
\hline matrícula padres & 0.640 & 0.02 \\
\hline matrícula crédito & -0.476 & 0.03 \\
\hline computador & 0.586 & 0.04 \\
\hline \multicolumn{3}{|l|}{ Razonamiento cuantitativo } \\
\hline matrícula padres & 0.4211 & 0.04 \\
\hline \multicolumn{3}{|l|}{ Inglés } \\
\hline edad & -0.161 & 0.00 \\
\hline estrato & 0.110 & 0.00 \\
\hline internet & 0.103 & 0.03 \\
\hline educación superior de la madre & 0.097 & 0.05 \\
\hline educación superior del padre & 0.104 & 0.04 \\
\hline \multicolumn{3}{|l|}{ Pensamiento crítico } \\
\hline edad & -0.037 & 0.00 \\
\hline$N^{\circ}$ de personas del grupo familiar & -0.047 & 0.01 \\
\hline estrato & 0.098 & 0.01 \\
\hline educación superior del padre & 0.178 & 0.01 \\
\hline \multicolumn{3}{|l|}{ Entendimiento interpersonal } \\
\hline género & -0.362 & 0.00 \\
\hline edad & -0.041 & 0.00 \\
\hline $\mathrm{N}^{\circ}$ de personas a cargo & 0.235 & 0.02 \\
\hline educación superior del padre & 0.157 & 0.04 \\
\hline ocupación independiente del padre & -0.181 & 0.01 \\
\hline \multicolumn{3}{|l|}{ Solución de problemas } \\
\hline edad & -0.042 & 0.00 \\
\hline estrato & 0.079 & 0.03 \\
\hline computador & 0.225 & 0.01 \\
\hline estudiante trabaja & 0.225 & 0.01 \\
\hline \multicolumn{3}{|l|}{ Comprensión lectora } \\
\hline edad & -0.037 & 0.00 \\
\hline
\end{tabular}

En Colombia existen diferentes posiciones sobre la influencia de aquellos factores que se asocian con mejores condiciones de vida del estudiante y de acceso a educación superior, como son el mayor nivel educativo de los padres y el al to estrato socioeconómico, pues algunos autores los señalan definitivos en los resultados del examen Saber Pro, tal es el caso del programa de medicina analizado durante $2009{ }^{16}$ y enfermería al inicio de los exámenes en $2003^{14}$, mientras que para otros investigadores estos no se asocian de manera significativa con los resultados obtenidos por los estudiantes en exámenes de estado, como fueron los estudios del programa de economía $\mathrm{a}^{13}$, donde fueron determinantes otros factores asociados con características de las universidades. AI respecto, esta investigación encontró que estos no se relacionaron en forma significativa con el desempeño del estudiante en los componentes del examen Saber Pro.

Dentro de las características de la IES, es importante mencionar que aquellas como el origen y el carácter académico, no se asociaron con los resultados obtenidos pero al comparar con la región a la cual pertenece la IES, se destaca que los estudiantes de las universidades de la región norte y sur del país obtuvieron los puntajes más bajos en la mayoría de los componentes como se muestra en la Figura 1, lo cual sugiere algunas deficiencias en la calidad de la educación en estas zonas del país, siendo pertinente profundizar un análisis de las condiciones actuales para las universidades de la región, en especial en la región norte donde existe un crecimiento de la oferta educativa y se registraron puntajes bajos en los exámenes en los diferentes programas de pregrado al inicio de las pruebas ECA ES. ${ }^{17}$

Se evidencia la necesidad de real izar un seguimiento continuo a los resultados en el examen Saber Pro a nivel nacional, que identifique aquellos factores sobre los cuales se puedan aplicar políticas de mejoramiento y de esta manera generar un impacto en la calidad y la evaluación de la educación superior en Colombia. 


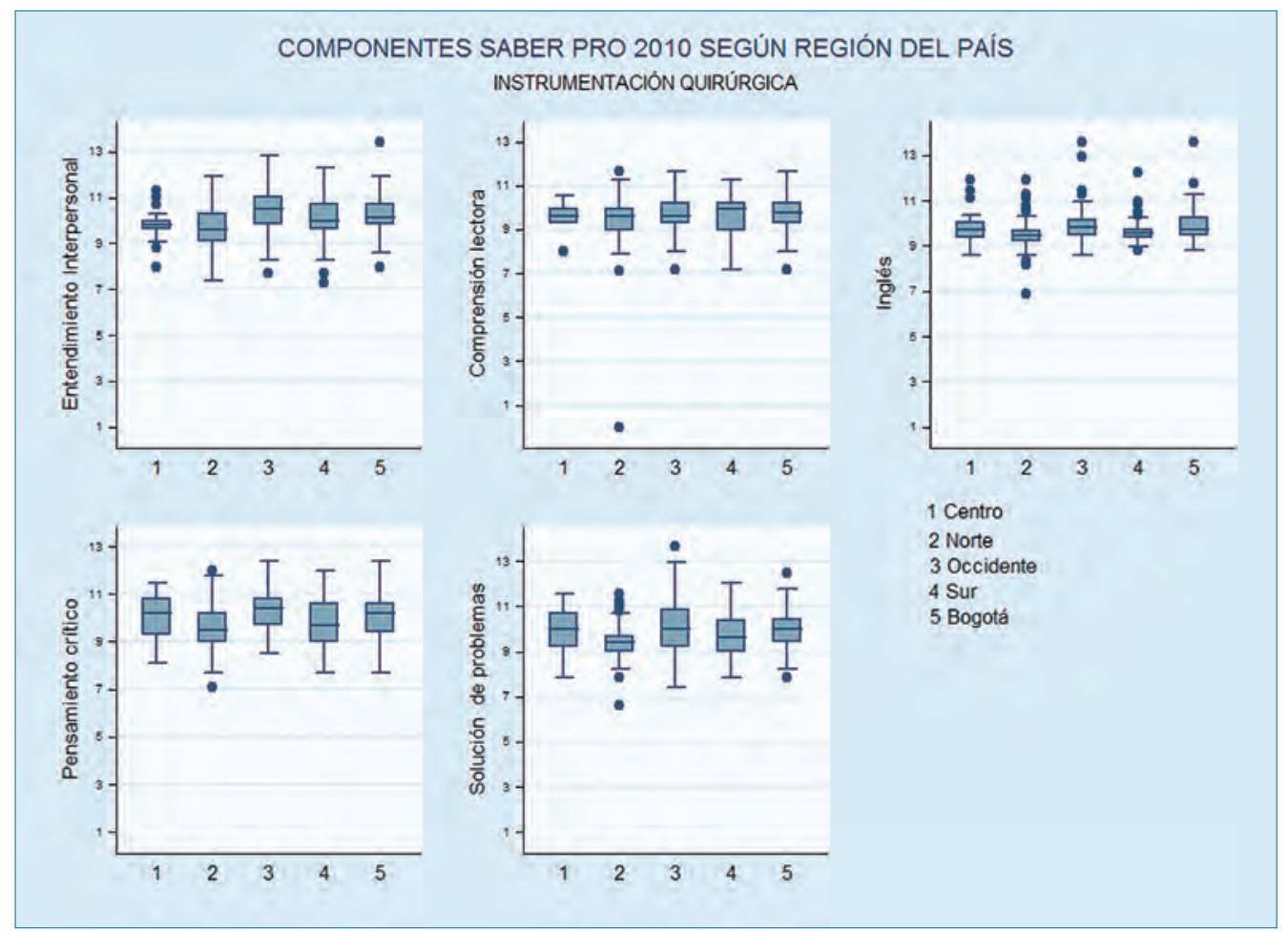

Figura I. Box plot de los puntajes obtenidos por los estudiantes de instrumentación quirúrgica en los componentes del examen Saber Pro según la región durante 2010.

\section{Referencias}

1. Colombia. Ministerio de Educación Nacional. Decreto 1781 de 2003, Por el cual se reglamentan los exámenes de estado de calidad de la educación superior ECAES de los estudiantes de los programas académicos de pregrado. Diario Oficial, 1781 (J un. 26 2003).

2. ICFES línea de tiempo en la transición del examen ICFES Saber Pro [Internet]. [consultado octubre 20 de 2013]. Disponible en: http://www.icfes.gov.co/examenes/component/docman/cat_view/10-saber-pro/22-informacion-general?!temid=

3. Díaz C. ECAES no es el indicador más clave: expertos aseguran que se ha convertido en presión para los estudiantes. Portafolio 2010 A gosto 31.

4. ¿Para qué sirven las pruebas Saber Pro? [Internet]. Bogotá: Diario El espectador. Vivir 8 de junio 2012. [consultado noviembre 20 de 2014]. Disponible en: http://www.elespectador.com/noticias/actualidad/vivir/sirven-pruebas-saber-proarticulo-352085.

5. Colombia. M inisterio de Educación Nacional. Decreto 3963 de 2009. Por el cual reglamenta el Examen de Estado de Calidad de la Educación Superior. Bogotá: El M inisterio; 2009.

6. Colombia. Congreso de la Republica. Ley 1324 de 2009 Por la cual se fijan parámetros y criterios para organizar el sistema de evaluación de resultados de la calidad de la educación, se dictan normas para el fomento de una cultura de la evaluación, en procura de facilitar la inspección y vigilancia del Estado y se transforma el ICFES. Diario Oficial, N 0. 47.409 (Julio 13 2009).

7. Instituto Colombiano para la Evaluación de la Educación ICFES. Guía de acceso a bases de datos ICFES. [Internet]. Bogotá: Instituto Colombiano para la Evaluación de la Educación ICFES; 2013 [consultado 20 Oct 2013]. Disponible en: http://www.icfes.gov.co/investigacion/component/docman/doc_view/208-guiageneral-ftp-febrero-2013-v3?!temid=

8. ICFES, exámenes de calidad de la educación superior en instrumentación quirúrgica. Guía de orientación de la prueba [Internet]. [consultado 2013 octubre 23]. Disponible en: http://www.colombiaaprende.edu.co/html/mediateca/1607/ articles-208990_recurso_1.pdf

9. Cortes S. A nálisis de las pruebas de estado y el desempeño académico. Estudiantes de instrumentación quirúrgica de la FUCS. Repert.M ed.Cir 2012; 21 (1): $58-62$.
10. Gaviria A, Barrientos J. Calidad de la educación y rendimiento académico en Bogotá [Internet]. [consultado 2013 octubre 20]; Disponible en: ftp://ftp.drivehq. com/cavasco/grupom/coyunturaN 024jorgehugo.pdf

11. Corsi L, Garcia M . Factores asociados a desempeños destacados y no destacados en las pruebas SA BER 1. [poster] en: III Seminario Internacional de Investigación sobre Calidad de la Educación. B ogotá; 2012.

12. Quispe J. Factores que influyen en el rendimiento académico y la deserción de los estudiantes de la Facultad de Ingeniería Económica de la UNA-PUNO, período 2009. Cuadernos de Educación y Desarrollo [Internet]. 2010 [consultado 23 de julio de 2014]; 2 (11) Disponible en: http://www.eumed.net/rev/ ced/11/jtq.htm

13. Valens M, Calidad de la educación superior en Colombia: un análisis multinivel con base en el ECAES de economía 2004. Revista sociedad y economía [Internet]. 2007 [consultado 3 de marzo de 2014]; 13: 132-154. Disponible en: http:// www.redalyc.org/pdf/996/99616721007.pdf

14. Cañón H, Cortes R, López M, Castellanos F, (2009). Rendimiento académico y desempeño en el Examen de Calidad de la Educación Superior (ECAES) de enfermería, año 2003. Investigación en Enfermería: Imagen y Desarrollo, 11(1), 67-80.

15. ICFES. Orientaciones para el examen de Estado de calidad de la educación superior SABER PRO (ECAES). prueba de competencias genéricas PDF. http://unilibrepereira.edu.co/A rchivos/A djuntos/60c5684fa5-ver-documento-sobre-prueba-de-competencias-genericas.pdf

16. Rodríguez V, Sepúlveda L, Rondón M . Gómez-Restrepo C. (2013). Impacto de las facultades de medicina y de los estudiantes sobre los resultados en la prueba nacional de calidad de la educación superior (SA BER PRO). Revista Colombiana de A nestesiología 41(3), 196-204.JA RA, Delia, et al. Factores influyentes en el rendimiento académico de estudiantes del primer año de medicina. En Anales de la Facultad de M edicina. U N M SM . Facultad de M edicina, 2008. p. 193-197.

17. Viloria De la Hoz, J. Educación superior en el Caribe colombiano: análisis de cobertura y calidad. [PDF]. Documentos de Trabajo sobre Economía Regional, B anco de la República, Centro de Estudios Económicos R egionales, marzo 2006. N 69. 